\title{
Imaging the oxygen-rich disk toward the silicate carbon star EU Andromedae ${ }^{\star}$
}

\author{
K. Ohnaka ${ }^{1}$ and D. A. Boboltz ${ }^{2}$ \\ 1 Max-Planck-Institut für Radioastronomie, Auf dem Hügel 69, 53121 Bonn, Germany \\ e-mail: kohnaka@mpifr-bonn.mpg.de \\ 2 United States Naval Observatory, 3450 Massachusetts Avenue, NW, Washington, DC 20392-5420, USA \\ e-mail: dboboltz@usno.navy.mil
}

Received 8 November 2007 / Accepted 8 December 2007

\begin{abstract}
Context. Silicate carbon stars are characterized by oxygen-rich circumstellar environments as revealed by prominent silicate emission, despite their carbon-rich photospheres. While the presence of a circumbinary disk or a disk around an unseen, low-luminosity companion has been suggested to explain the peculiar dust chemistry, the origin of silicate carbon stars is still a puzzle to date.

Aims. We present multi-epoch, high-angular resolution observations of $22 \mathrm{GHz} \mathrm{H_{2 }} \mathrm{O}$ masers toward the silicate carbon star EU And to probe the spatio-kinematic distribution of oxygen-rich material.

Methods. EU And was observed at three epochs (maximum time interval of 14 months) with the Very Long Baseline Array (VLBA). Results. Our VLBA observations of the $22 \mathrm{GHz} \mathrm{H} \mathrm{H}_{2} \mathrm{O}$ masers have revealed that the maser spots are distributed along a straight line across $\sim 20$ mas, with a slight hint of an S-shaped structure. The observed spectra show three prominent velocity components at $V_{\mathrm{LSR}}=-42,-38$, and $-34 \mathrm{~km} \mathrm{~s}^{-1}$, with the masers in SW redshifted and those in NE blueshifted. The maser spots located in the middle of the overall distribution correspond to the component at $V_{\mathrm{LSR}}=-38 \mathrm{~km} \mathrm{~s}^{-1}$, which approximately coincides with the systemic velocity. These observations can be interpreted as either an emerging helical jet or a disk viewed almost edge-on (a circumbinary or circum-companion disk). However, the outward motion measured in the VLBA images taken 14 months apart is much smaller than what is expected from the jet scenario. Furthermore, the mid-infrared spectrum obtained with the Spitzer Space Telescope indicates that the $10 \mu \mathrm{m}$ silicate emission is optically thin and the silicate grains are of sub-micron size. This lends support to the presence of a circum-companion disk, because an optically thin circumbinary disk consisting of such small grains would be blown away by the intense radiation pressure of the primary (carbon-rich) star. If we assume Keplerian rotation for the circum-companion disk, the mass of the companion is estimated to be $0.5-0.8 M_{\odot}$. We also identify $\mathrm{CO}_{2}$ emission features at 13-16 $\mu \mathrm{m}$ in the Spitzer spectrum of EU And-the first unambiguous detection of $\mathrm{CO}_{2}$ in silicate carbon stars.
\end{abstract}

Key words. radio lines: stars - techniques: interferometric - stars: circumstellar matter - stars: carbon stars: AGB and post-AGB - stars: individual: EU And

\section{Introduction}

Oxygen-rich circumstellar material (i.e., silicate and/or $\mathrm{Al}_{2} \mathrm{O}_{3}$ ) is usually associated with M-type asymptotic giant branch (AGB) stars, reflecting their photospheric chemical composition. Surprisingly, however, carbon stars showing silicate emission (so-called "silicate carbon stars") were discovered by IRAS (Little-Marenin 1986; Willems \& de Jong 1986). The subsequent detection of $\mathrm{H}_{2} \mathrm{O}$ and $\mathrm{OH}$ masers toward some of the silicate carbon stars, if not all, confirmed the oxygen-rich nature of the circumstellar material (e.g., Nakada et al. 1987, 1988; Benson \& Little-Marenin 1987; Little-Marenin et al. 1988; Barnbaum et al. 1991; Engels 1994). On the other hand, optical spectroscopic studies show that ${ }^{12} \mathrm{C} /{ }^{13} \mathrm{C}$ ratios in silicate carbon stars are as low as 4-5 and thus they are classified as "J-type" carbon stars (e.g., Ohnaka \& Tsuji 1999), which is difficult to explain by the standard stellar evolution theory.

One currently accepted hypothesis suggests that silicate carbon stars have a low-luminosity companion (possibly a mainsequence star or a white dwarf) and that oxygen-rich material

* This work is based [in part] on observations made with the Spitzer Space Telescope, which is operated by the Jet Propulsion Laboratory, California Institute of Technology under a contract with NASA. was shed by mass loss when the primary star was an oxygenrich giant. The oxygen-rich material is stored in a circumbinary disk even after the primary star becomes a carbon star (Morris 1987; Lloyd-Evans 1990). Alternatively, Yamamura et al. (2000) propose that the oxygen-rich material is stored in a circumstellar disk around the companion. In this scenario, the observed silicate emission cannot originate directly from the circumcompanion disk heated by the companion itself, because its luminosity is too low. Instead, Yamamura et al. (2000) argue that radiation pressure from the primary, carbon-rich, AGB star drives and heats an outflow from the circum-companion disk. The observed silicate emission originates from this outflow.

Our recent $N$-band ( $8-13 \mu \mathrm{m})$ spectro-interferometric observations of the silicate carbon star IRAS 08002-3803 using the ESO's Very Large Telescope Interferometer (VLTI) have spatially resolved the dusty environment of a silicate carbon star for the first time, and our radiative transfer modeling shows that the $N$-band visibilities can be fairly explained by an optically thick circumbinary disk in which small $(\sim 0.1 \mu \mathrm{m})$, amorphous silicate and a second grain species - amorphous carbon, large silicate grains $(\sim 5 \mu \mathrm{m})$, or metallic iron - coexist (Ohnaka et al. 2006). The $N$-band visibilities observed on another silicate carbon star IRAS 18006-3213 show a wavelength dependence very similar 
to IRAS 08002-3803, suggesting that IRAS 18006-3213 also has a circumbinary disk (Deroo et al. 2007).

Some evidence of long-lived disks around silicate carbon stars has been discovered by radio observations as well. Jura \& Kahane (1999) detected narrow CO $(J=2-1,1-0)$ emission lines toward two silicate carbon stars EU And and BM Gem, which indicate the presence of a reservoir of orbiting gas. Szczerba et al. (2006) and Engels (priv. comm.) obtained high-resolution $22 \mathrm{GHz} \mathrm{H}_{2} \mathrm{O}$ maser maps toward the silicate carbon star V778 Cyg using MERLIN and VLBA, respectively. The $\mathrm{H}_{2} \mathrm{O}$ maser distributions are linearly aligned with a slightly S-shaped structure, which Szczerba et al. (2006) and Babkovskaia et al. (2006) interpret as a warped circumcompanion disk viewed almost edge-on. Therefore, as discussed in Yamamura et al. (2000) and Ohnaka et al. (2006), there may be two classes of silicate carbon stars: systems with optically thick circumbinary disks and those with circum-companion disks.

While direct detection of companions of silicate carbon stars is still difficult due to the huge luminosity contrast $\left(\sim 10^{4} L_{\odot}\right.$ and $\lesssim 1 L_{\odot}$ for the primary star and the companion, respectively), Izumiura (2003) and Izumiura et al. (2007) detected blue continuum emission at $\$ 4000 \AA$ and Balmer lines showing $P$ Cygni profiles with an outflow velocity of $\gtrsim 400 \mathrm{~km} \mathrm{~s}^{-1}$ toward BM Gem, which strongly suggests the presence of an accretion disk around an unseen companion.

Despite this observational progress, the formation mechanisms of the circumbinary or circum-companion disks as well as the peculiar photospheric chemical composition of silicate carbon stars are little understood. Radio interferometry observations of the maser emission produced by oxygen-bearing molecules provide us with an excellent opportunity to study the spatio-kinematic distribution of oxygen-rich gas around silicate carbon stars. In this paper, we present the results of multi-epoch, high-resolution observations of the $22 \mathrm{GHz} \mathrm{H}_{2} \mathrm{O}$ masers toward the silicate carbon star EU And.

\section{Observations}

The $\mathrm{H}_{2} \mathrm{O}$ maser emission associated with EU And $(\alpha=$ $23^{\mathrm{h}} 19^{\mathrm{m}} 58.8814^{\mathrm{s}}, \delta=47^{\circ} 14^{\prime} 34.567^{\prime \prime}$, J2000.0, Epoch 2000.0, NOMAD Catalog, Zacharias et al. 2003) ${ }^{1}$ was observed at a rest frequency of $22.23508 \mathrm{GHz}$, using the Very Long Baseline Array (VLBA) operated by the National Radio Astronomy Observatory $(\mathrm{NRAO})^{2}$. The observations were carried out over three epochs spanning approximately 14 months: 2005 November 5, 2006 March 19, and 2007 January 13. VLBA's 10 antennas were used at the first and second epochs, while only 9 antennas could be used at the third epoch due to a technical problem of the St. Croix station. For each 5-h epoch, EU And and two extragalactic calibrator sources (3C 454.3 and $\mathrm{J} 2322+5057$ ) were observed. We used the technique of rapid switching between the target source and the nearby phase reference source, $\mathrm{J} 2322+5057$, in order to remove residual phase offsets in the target source due to the atmosphere. The data were

\footnotetext{
1 This is offset by $32^{\prime \prime}$ from the position used for the VLA observation on 1990 June 2 by Colomer et al. (2000), who detected no $\mathrm{H}_{2} \mathrm{O}$ maser toward EU And. The negative detection may have been due to this positional offset, although it is still possible that the $\mathrm{H}_{2} \mathrm{O}$ masers were indeed absent in June 1990.

2 The National Radio Astronomy Observatory is a facility of the National Science Foundation operated under cooperative agreement by Associated Universities, Inc.
}

recorded in dual circular polarization with the 8- $\mathrm{MHz}$ band centered on a local standard of rest (LSR) velocity of $-36.0 \mathrm{~km} \mathrm{~s}^{-1}$. The system temperatures and the sensitivities were of the order of $100 \mathrm{~K}$ and $10 \mathrm{Jy} \mathrm{K}^{-1}$, respectively, for all three epochs.

The data were correlated at the VLBA correlator in Socorro, New Mexico, which produced auto- and cross-correlation spectra with 512 channels, corresponding to a channel spacing of $15.63 \mathrm{kHz}\left(0.22 \mathrm{~km} \mathrm{~s}^{-1}\right)$. For the calibration of the correlated data, we followed the standard reduction procedures for VLBA spectral line experiments using the Astronomical Image Processing System (AIPS) maintained by NRAO. A bandpass calibration was carried out using intermittent (every $30 \mathrm{~min}$ ) scans on 3C 453.3. A fringe fit was performed on the phasereference source in order to remove effects due to instrumentation and atmosphere on the phase not removed in the correlation. The resulting residual phase delays, phase rates, and phases were applied to the target source. At this point a preliminary image cube was produced for EU And. Because the signal-to-noise in these images was not optimal, we performed a second fringe fit on a strong reference maser feature (at $V_{\mathrm{LSR}}=$ $-34 \mathrm{~km} \mathrm{~s}^{-1}$ ) in the spectrum of EU And and applied the residual phase rates to the target. Finally, an iterative self-calibration and imaging procedure was performed on the reference channel and the solutions for the residual phase and amplitude corrections were applied to all spectral channels. Images with $1024 \times 1024$ pixels $(61 \times 61$ mas $)$ were produced for all velocity channels between $V_{\mathrm{LSR}}=-24$ and $-52 \mathrm{~km} \mathrm{~s}^{-1}$ using beam sizes of $0.71 \times 0.29$ mas, $0.73 \times 0.36$ mas, and $0.82 \times 0.31$ mas for the first, second, and third epochs, respectively. Typical rms off-source noise in the final images is $7-10 \mathrm{mJy} \mathrm{beam}^{-1}$.

Maser components were identified, and their positions were measured by fitting the emission in the final images of each spectral channel with two-dimensional (2D) Gaussians using the AIPS task SAD. The errors of the positions (within an epoch) are 10-20 $\mu$ as for the strong maser features and approach $\sim 50 \mu$ as for the weaker features. The absolute astrometric information obtained by phase-referencing is lost in the above self-calibration procedure. To restore this information to the final component positions, we fit 2D Gaussians to the strongest maser feature (assumed to be the same for all three epochs at $V_{\mathrm{LSR}}=-34 \mathrm{~km} \mathrm{~s}^{-1}$ ) prior to any self-calibration. The resulting shifts were applied to all maser components identified in the final images. The absolute positions of the strongest maser obtained for the first, second, and third epochs are $\left(23^{\mathrm{h}} 19^{\mathrm{m}} 58.8822^{\mathrm{s}}\right.$, $\left.47^{\circ} 14^{\prime} 34.550^{\prime \prime}\right), \quad\left(23^{\mathrm{h}} 19^{\mathrm{m}} 58.8823^{\mathrm{s}}, \quad 47^{\circ} 14^{\prime} 34.548^{\prime \prime}\right)$, and $\left(23^{\mathrm{h}} 19^{\mathrm{m}} 58.8824^{\mathrm{s}}, 47^{\circ} 14^{\prime} 34.546^{\prime \prime}\right)$, respectively (all in J2000.0).

The accuracy of the absolute position of the strongest maser feature is primarily limited by errors resulting from the transfer of the residual phase corrections from the extragalactic reference source to the target source and by the error in the absolute position of the reference source itself. In order to estimate the error involved in the transfer of the residual phase corrections, we essentially reversed the procedure used to determine the absolute position of the target maser source. We applied the residual phase solutions resulting from the self-calibration on the target source (strongest maser at $V_{\mathrm{LSR}}=-34 \mathrm{~km} \mathrm{~s}^{-1}$ ) to the extragalactic reference source. We then imaged the reference source and performed a 2D Gaussian fit to determine its position. The separation between the positions of the target/calibrator pair (i.e., the arc length) was computed for 1) both sources calibrated with the extragalactic reference source solutions, and 2) both sources calibrated with the target source solutions. The difference between the arc lengths yields an estimate of the error involved in the phase transfer. The computation of the arc lengths is 

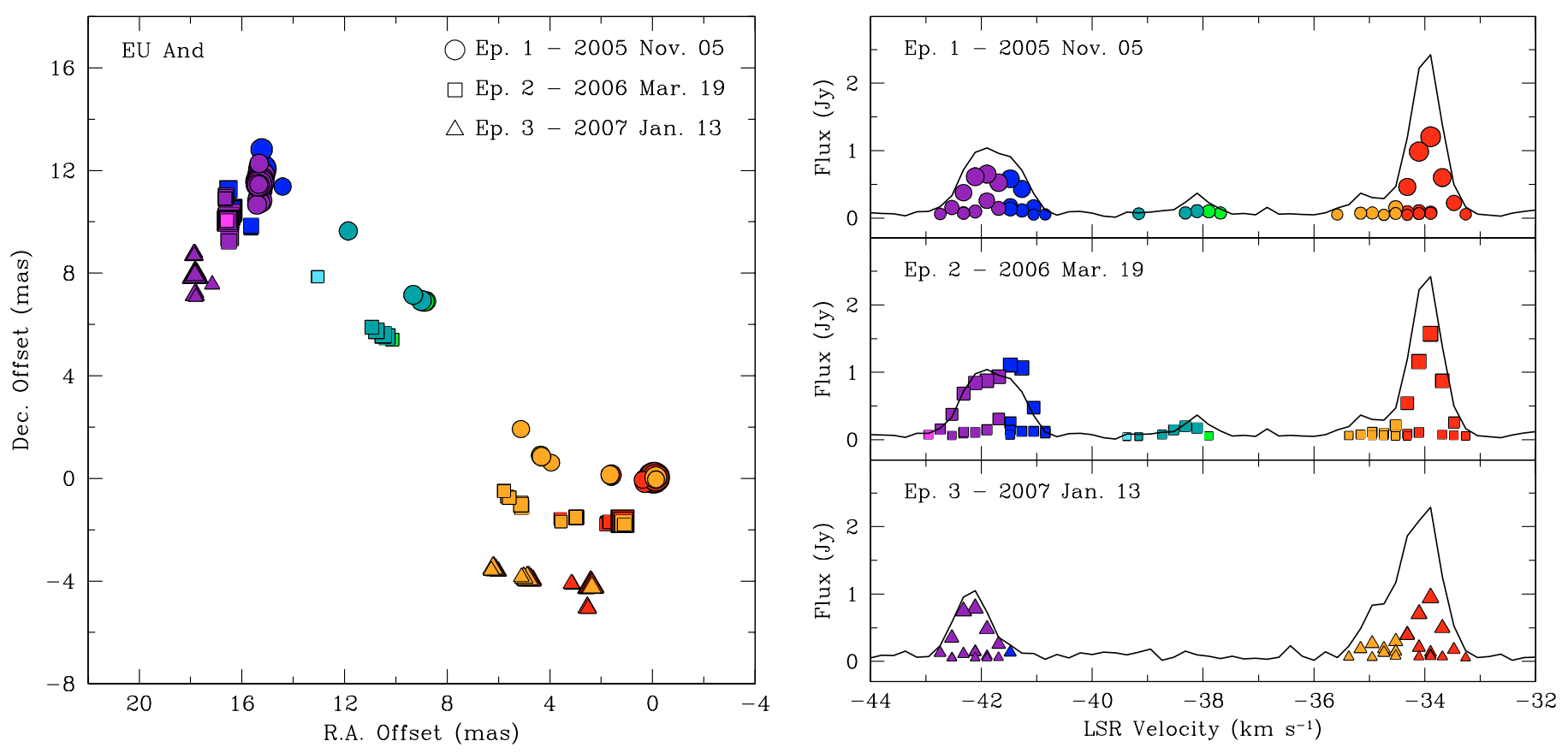

Fig. 1. $22 \mathrm{GHz} \mathrm{H}_{2} \mathrm{O}$ masers observed toward EU And with VLBA at three epochs. The positions and velocities of the $\mathrm{H}_{2} \mathrm{O}$ maser spots are derived by two-dimensional Gaussian fitting as described in Sect. 2. Left: spatial distribution of the $\mathrm{H}_{2} \mathrm{O}$ masers with point-color representing the corresponding velocity bin in the spectrum and point-size proportional to the logarithm of the maser flux density. The origin of RA and Dec offsets is $\left(23^{\mathrm{h}} 19^{\mathrm{m}} 58.8822^{\mathrm{s}}, 47^{\circ} 14^{\prime} 34.550^{\prime \prime}, \mathrm{J} 2000.0\right)$. The maser spots observed at three epochs are positionally offset because of proper motion and parallax. Right: Spectra formed by plotting maser component flux density versus LSR velocity, color-coded according to maser velocity. The solid lines represent vector-averaged cross-power spectra on the Los Alamos-Pie Town VLBA baseline. (A color version of this figure is available in the electronic edition.)

unnecessary in experiments specifically designed for astrometry where a second extragalactic check source is typically observed. This procedure was repeated for all three epochs and the standard deviation of the difference in arc lengths was found to be 0.12 mas. This error in the phase transfer compares favorably with the error in the absolute position of the reference source itself, which is a defining source of the International Celestial Reference Frame (ICRF) with an error of 0.26 mas (Fey et al. 2004). The total error in the absolute position of the strongest maser feature is estimated from the root-sum-square of the two errors described above to be $\sim 0.3$ mas.

\section{Results}

Figure 1 shows the spatio-kinematic distributions (left panel) and spectra (right panels) of the $\mathrm{H}_{2} \mathrm{O}$ masers toward EU And as observed over the three epochs. At all epochs, the $\mathrm{H}_{2} \mathrm{O}$ maser spectra are characterized by two strong peaks at $V_{\mathrm{LSR}}=$ $-34 \mathrm{~km} \mathrm{~s}^{-1}$ and $-42 \mathrm{~km} \mathrm{~s}^{-1}$. In the first and second epochs, weak features were also detected at $-38 \mathrm{~km} \mathrm{~s}^{-1}$, approximately in the middle of the two peaks. The velocity of these weak maser features is in agreement with that of the narrow $\mathrm{CO}$ lines $\left(\sim-37 \mathrm{~km} \mathrm{~s}^{-1}\right)$ measured by Jura \& Kahane (1999). The velocity of the weak maser features also coincides with the radial velocities derived from the optical spectra, which represent the velocity of the primary carbon-rich AGB star (Barnbaum 1991). However, as discussed below, the putative companion, instead of the primary star, is more likely to be located at the position of these weak maser components. We note that while the spectra taken over 14 months appear rather stable, the $\mathrm{H}_{2} \mathrm{O}$ masers toward EU And exhibit remarkable temporal variations in strength and velocity. For example, Little-Marenin (1988) detected maser components near $V_{\mathrm{LSR}}=-30 \mathrm{~km} \mathrm{~s}^{-1}$ as strong as $8 \mathrm{Jy}$, which are non-existent in our VLBA data.

The $\mathrm{H}_{2} \mathrm{O}$ masers at all three epochs are linearly aligned across $\sim 20$ mas, with a slight hint of an S-shaped structure. As explained in Sect. 2, the offsets among the epochs shown in Fig. 1 are real and represent the proper motion and parallax. In fact, the slight curvature seen in the direction of the positional displacement over the three epochs, which is discernible particularly for the strong features (the one at $(0,0)$ at the first epoch), shows the annual parallax component. The observed S-shaped structure can be interpreted as an edge-on disk or an emerging helical jet. Such a nearly linear distribution of the $\mathrm{H}_{2} \mathrm{O}$ masers is similar to those observed toward the so-called water fountain sources (e.g., Imai et al. 2002; Boboltz \& Marvel 2007, and references therein), which show well-collimated, fast jets of $\mathrm{H}_{2} \mathrm{O}$ masers. However, the outflow velocities of the $\mathrm{H}_{2} \mathrm{O}$ masers toward the water fountain sources are much higher $(\sim 60-$ $\left.150 \mathrm{~km} \mathrm{~s}^{-1}\right)$ than that observed toward EU And $\left(\sim 5 \mathrm{~km} \mathrm{~s}^{-1}\right)$, although the projection effect is uncertain.

In order to examine the jet scenario, we measured the increase of the separation (arc length) between the masers associated with the two highest peaks of emission in the spectrum. Since a single maser spans multiple channels in the spectrum, we performed a flux density squared weighted average over velocity and position prior to measuring the separations between the two peak features. The difference in the arc lengths between the first and third epochs is $\sim 0.3$ mas. Using this measurement, we estimate the velocity $V$ and the inclination angle $i$ (angle toward us out of the plane of the sky) of a water-fountain-like outflow for EU And as follows. The observed velocity separation of the two strongest masers is $8 \mathrm{~km} \mathrm{~s}^{-1}$, which means that $2 V \sin i=8 \mathrm{~km} \mathrm{~s}^{-1}$. The spatial separation of the two masers 


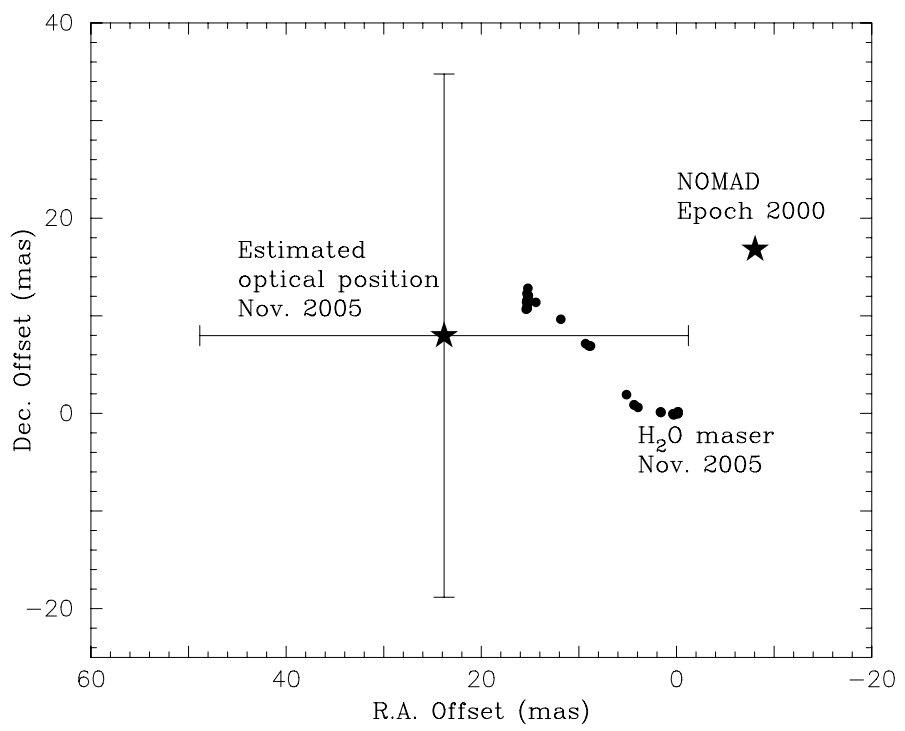

Fig. 2. The optical position of EU And in 2000 (NOMAD catalog) and the expected optical position at the first epoch of the VLBA observations (Nov. 2005). The error bars are the sum of the uncertainties of the absolute position and the proper motion (see Sect. 4).

increases by $2 V \cos i \times \Delta t / d=0.3$ mas, where $\Delta t$ is the time interval between the first and third epochs (14 months), and $d$ is the distance of EU And. With distances of 1.5-2.6 kpc adopted (Jura \& Kahane 1999; Engels 1994), we obtain $V=4.1-$ $4.3 \mathrm{~km} \mathrm{~s}^{-1}$ and $i=68-77^{\circ}$. These outflow velocities are much lower than those observed toward the water-fountain sources, making the helical jet interpretation unfavorable, although the distance of EU And may be remarkably larger than the above estimates and/or the outflow velocity of EU And may indeed be so low due to some driving mechanism different from that in the water-fountain sources. A further long-term monitoring as well as a measurement of proper motion with VLBA would be necessary to settle this issue.

The $\mathrm{H}_{2} \mathrm{O}$ maser distributions observed toward EU And are similar to that observed toward V778 Cyg by Szczerba et al. (2006) and Engels (priv. comm.). Our observations of another silicate carbon star IRAS 07204-1032 using VLBA and the Very Large Array (VLA) have also revealed a similar spectrum and spatial distribution of the $\mathrm{H}_{2} \mathrm{O}$ masers (Boboltz et al. in prep.). The expectation of observing linear distributions of masers toward the three silicate carbon stars imaged to date may seem statistically unlikely, given a random distribution of disk inclination angles. However, it is quite possible that this is due to a selection effect in which masers are preferentially detected in objects with the most favorable geometry for maser amplification (i.e., nearly edge-on disks). A survey of more objects with VLA and VLBA would be useful for obtaining a definitive answer to the interpretation of the masers toward silicate carbon stars.

\section{Discussion}

Although our $\mathrm{H}_{2} \mathrm{O}$ maser maps of EU And lend support to the presence of an edge-on disk, these observations alone cannot clarify whether the disk is located around the carbonrich primary star (circum-primary disk) or the whole binary system (circumbinary disk) or an unseen companion (circumcompanion disk). The optical position of EU And, which corresponds to that of the primary star, is $(\alpha, \delta)=\left(23^{\mathrm{h}} 19^{\mathrm{m}} 58.8814^{\mathrm{s}}\right.$, $\left.+47^{\circ} 14^{\prime} 34.567^{\prime \prime}, \mathrm{J} 2000.0\right)$ with proper motion of $\left(\mu_{\alpha}, \mu_{\delta}\right)=$ $(5.4 \pm 1.7,-1.5 \pm 2.0)$ mas $_{\mathrm{yr}^{-1}}$ (NOMAD catalog, Zacharias et al. 2003). The position of the primary star expected for the first epoch, which is shown in Fig. 2, appears to be offset from the $\mathrm{H}_{2} \mathrm{O}$ masers. However, the uncertainty of the absolute position ( \pm 15 mas, Zacharias et al. 2004) as well as that of the proper motion is too large to examine whether the primary star is located at the center of the $\mathrm{H}_{2} \mathrm{O}$ masers or indeed offset from the maser distribution.

\subsection{Constraints from the mid-infrared spectrum}

On the other hand, as Yamamura et al. (2000) argue, a circumprimary or circumbinary disk would be unstable against the intense radiation pressure from the primary carbon-rich star $\left(\sim 10^{3}-10^{4} L_{\odot}\right)$, if the disk is entirely optically thin. In order to examine whether or not the silicate dust around EU And is optically thin, we obtained its mid-infrared spectrum from the data archive of the Spitzer Space Telescope (Werner et al. 2004). EU And was observed on 2004 December 9 (Program ID: P03235, P.I.: C. Waelkens) with the InfraRed Spectrograph ${ }^{3}$ (IRS, Houck et al. 2004) in the Short-High (SH) and LongHigh (LH) modes with a spectral resolution of $\sim 600$. We downloaded the Basic Calibrated Data (BCD) processed with the S153 pipeline and extracted the spectrum using SMART v.6.2.5 (Higdon et al. 2004) ${ }^{4}$. Figure 3 shows the Spitzer/IRS spectrum of EU And (no sky subtraction was performed) together with the spectra of the silicate carbon star V778 Cyg and the oxygen-rich AGB star $o$ Cet obtained with the Short Wavelength Spectrometer (SWS) onboard the Infrared Space Observatory (ISO). As Yamamura et al. (2000) show, the $10 \mu \mathrm{m}$ silicate features of the latter two stars indicate that the silicate emission is optically thin and silicate grains are of sub-micron size. Obviously, the spectrum of EU And closely resembles those of V778 Cyg and $o$ Cet. A comparison with the IRAS $12 \mu \mathrm{m}$ flux of EU And and its Spitzer/IRS spectrum reveals that the flux level has little changed for the last 21 years, and EU And is similar to V778 Cyg in this aspect as well. This means that the silicate emission of EU And is optically thin with sub-micron grain sizes, rendering the possibility of a circum-primary or circumbinary disk unlikely. Therefore, the disk-like structure discovered by the $\mathrm{H}_{2} \mathrm{O}$ masers is likely to represent a circum-companion disk.

The predominance of sub-micron-sized grains appears to contradict the conclusion of Jura \& Kahane (1999) that the grain size around EU And should be as large as $\geq 0.2 \mathrm{~cm}$ to be gravitationally bound against radiation pressure. However, they assumed a circum-primary or circumbinary disk around the central star with a luminosity of $10^{4} L_{\odot}$. In the case of a disk around a low-luminosity companion $\left(\sim 1 L_{\odot}\right)$, grains as small as $0.2 \mu \mathrm{m}$ are stable against the radiation pressure of the companion, although this does not entirely exclude the presence of larger grains due to grain growth in dense regions (e.g., mid-plane) of the circum-companion disk.

In the Spitzer/IRS spectrum of EU And shown in Fig. 3, we can identify the $\mathrm{CO}_{2}$ emission features. To illustrate this identification, we also show the (scaled) absorption cross sections of ${ }^{12} \mathrm{CO}_{2}$ and ${ }^{13} \mathrm{CO}_{2}$, which were calculated using the $\mathrm{CO}_{2}$ line list

3 The IRS was a collaborative venture between Cornell University and Ball Aerospace Corporation funded by NASA through the Jet Propulsion Laboratory and Ames Research Center.

${ }^{4}$ SMART was developed by the IRS Team at Cornell University and is available through the Spitzer Science Center at Caltech. 

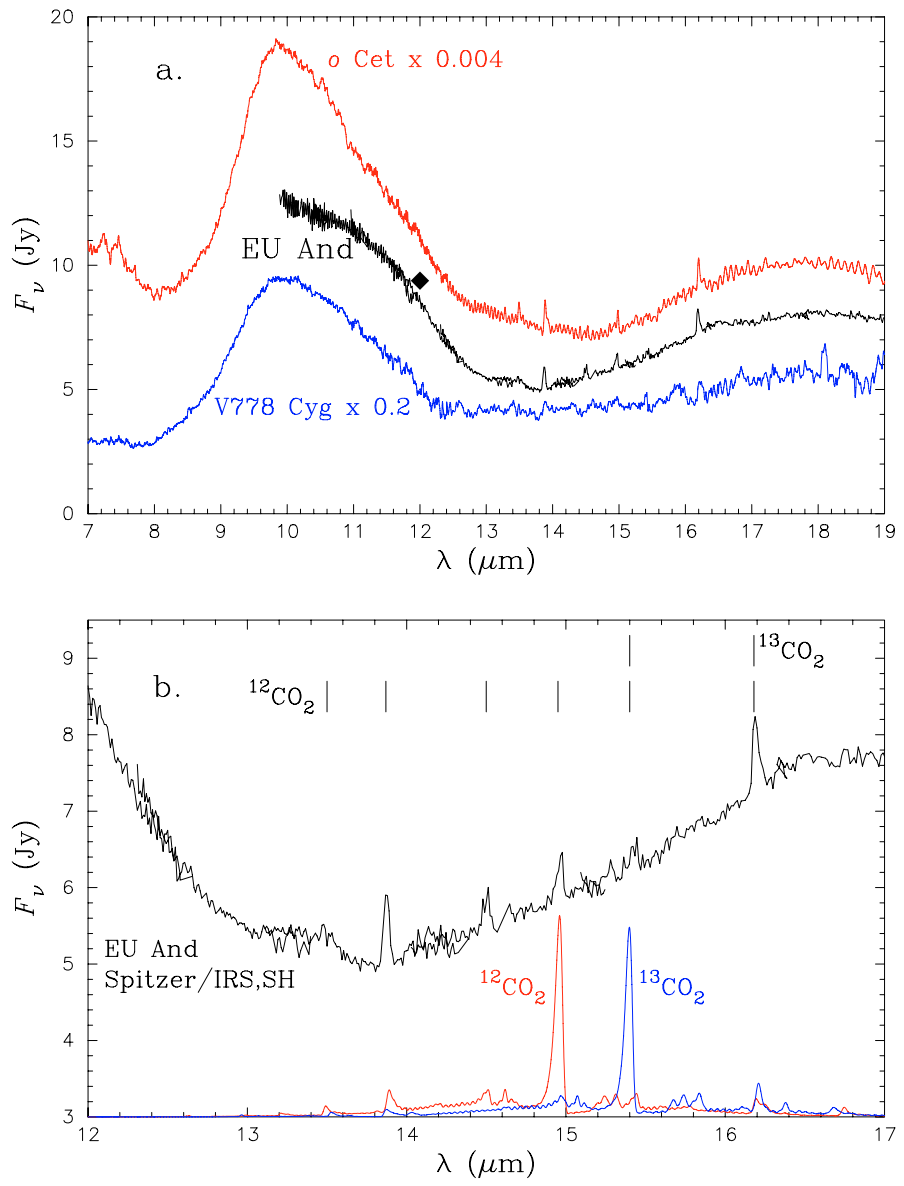

Fig. 3. a) Spitzer/IRS spectrum of EU And, together with the scaled ISO/SWS spectra of V778 Cyg and $o$ Cet. The diamond represents the IRAS $12 \mu$ m flux of EU And. b) $\mathrm{CO}_{2}$ emission features identified in the Spitzer/IRS spectrum of EU And are marked with the ticks. The scaled absorption cross section of ${ }^{12} \mathrm{CO}_{2}$ and ${ }^{13} \mathrm{CO}_{2}$ for $1000 \mathrm{~K}$ are also shown.

of the HITRAN database (Rothman et al. 2005) for $1000 \mathrm{~K}$ (the column densities of the both isotopic species are set to be equal). The $\mathrm{CO}_{2}$ features are clearly seen in the ISO/SWS spectrum of the oxygen-rich star $o$ Cet, and also marginally in V778 Cyg. Yamamura et al. (2000) could only tentatively identify these $\mathrm{CO}_{2}$ features in V778 Cyg because of the low $\mathrm{S} / \mathrm{N}$ ratio, and the case of EU And is the first unambiguous detection of $\mathrm{CO}_{2}$ toward silicate carbon stars. This is another piece of evidence for oxygenrich gas around silicate carbon stars, and the mid-infrared $\mathrm{CO}_{2}$ features will enable us to estimate the $\mathrm{CO}_{2}$ density and temperature if the disk structure is more tightly constrained in the future. It would also be possible to derive the ${ }^{12} \mathrm{C} /{ }^{13} \mathrm{C}$ ratio of the circumstellar gas from an analysis of the mid-infrared ${ }^{12} \mathrm{CO}_{2}$ and ${ }^{13} \mathrm{CO}_{2}$ features, which would shed new light on the origin of the abnormally low ${ }^{12} \mathrm{C} /{ }^{13} \mathrm{C}$ ratios in silicate carbon stars.

It is worth noting that $o$ Cet (Mira AB), whose mid-infrared spectrum closely resembles that of EU And, is a well-known binary system (separation $\sim 0$.'5) with a main-sequence or white dwarf companion. The high-resolution mid-infrared images of Mira AB recently obtained by Ireland et al. (2007) suggest that the edge of an accretion disk around Mira B (companion) is heated by Mira A (the primary star at the AGB). As Izumiura et al. (2007) suggest, this may resemble the circumstellar environment of some - if not all - silicate carbon stars (including EU And). Unlike silicate carbon stars, the circum-companion disk around Mira B consisting of silicate is embedded in the

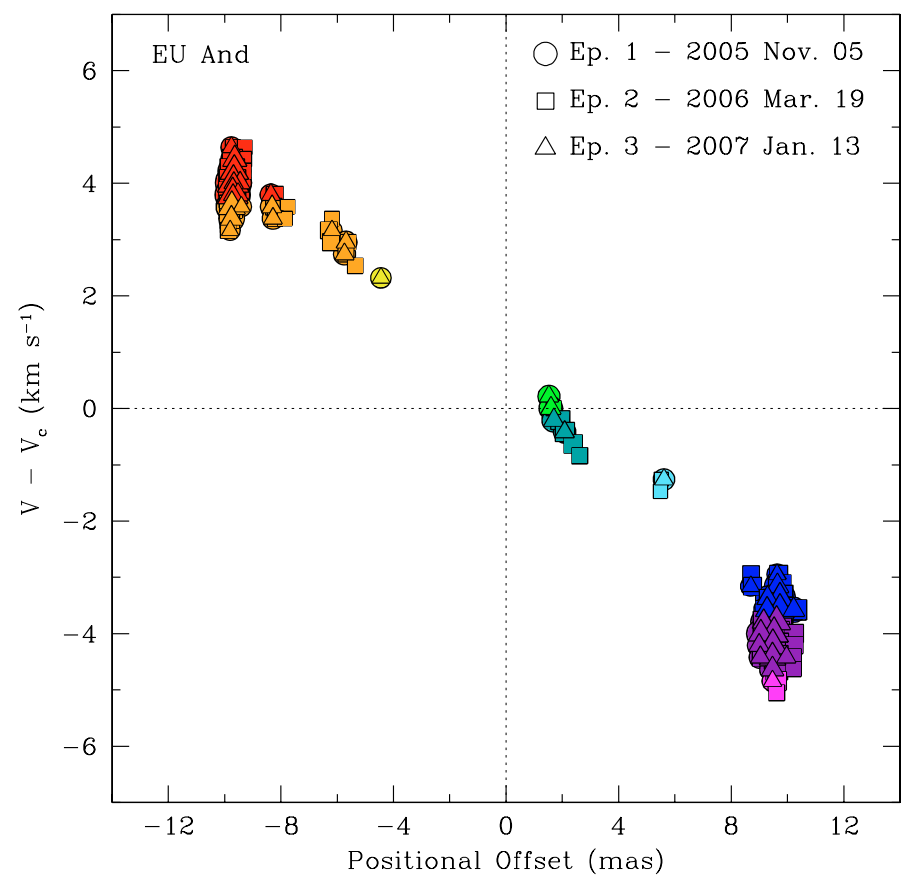

Fig. 4. The $p-V$ diagram for the $\mathrm{H}_{2} \mathrm{O}$ masers toward EU And. The center position and velocity were derived as described in Sect. 4.2. The center position (for the first epoch) is located at $(7.7,5.9)$ mas in the left panel of Fig. 1, while the center velocity $V_{\mathrm{c}}$ is $-37.9 \mathrm{~km} \mathrm{~s}^{-1}$. The circles, squares, and triangles represent the data taken at the first, second, and third epochs, respectively, with the velocities represented by the colors as in Fig. 1. (A color version of this figure is available in the electronic edition.)

oxygen-rich outflow from Mira A, which masks the mid-infrared spectroscopic signature of the circum-companion disk.

\subsection{Nature of the companion}

In order to draw a $p-V$ diagram for the observed masers toward EU And, we determined the center of the overall spatial distribution by taking the mid-point of a line connecting the mean positions of the redshifted and blueshifted masers at each epoch. The center velocity was also derived by halving the difference between the mean velocities of the redshifted and blueshifted masers. The resulting $p-V$ diagram for EU And is shown in Fig. 4. It is very similar to that derived for V778 Cyg by Szczerba et al. (2006), although the number of maser spots is much smaller than for V778 Cyg. Both objects show a linear part with very large gradients at both ends of the velocity range. This is consistent with the presence of a Keplerian disk, although it is not definitive evidence. If we assume that the (circum-companion) disk is in Keplerian rotation, and the center of the disk is located in the middle of the maser distribution at $V_{\mathrm{LSR}}=-37.9 \mathrm{~km} \mathrm{~s}^{-1}$, we can estimate the mass of the companion at the disk center. As shown in Pestalozzi et al. (2004), maser emission observed toward an edge-on disk has three maxima - on the line of sight toward the disk center and near the lines of sight tangential to the outer edge of the disk. If the strong $\mathrm{H}_{2} \mathrm{O}$ maser components to $\mathrm{NE}$ and $\mathrm{SW}$ correspond to the maxima near the outer edge of the disk, it follows that the Keplerian velocity is $\sim 5 \mathrm{~km} \mathrm{~s}^{-1}$ at a radius of 10 mas $=10 \times$ $(d / \mathrm{kpc}) \mathrm{AU}$, where $d$ is the distance of EU And. This translates into $0.3(d / \mathrm{kpc}) M_{\odot}$ for the central mass $\left(0.5-0.8 M_{\odot}\right.$ for $d=$ $1.5-2.6 \mathrm{kpc})$. This mass would be reasonable for an unevolved main-sequence star or a white dwarf. 
It should be noted, however, that the strong maser components at -42 and $-34 \mathrm{~km} \mathrm{~s}^{-1}$ may not represent the outer edge of the disk. As mentioned above, the strong maser components at $\sim-30 \mathrm{~km} \mathrm{~s}^{-1}$ were detected by Little-Marenin et al. (1988), but the lack of spatial information does not allow us to take these observations into account in estimating the mass of the secondary star. Moreover, there may be masers fainter than the VLBA's detection limit and/or more diffuse emission which would be resolved out by VLBA. Therefore, the above estimate of the companion mass based on Keplerian rotation is a lower limit. The estimate of the companion mass also depends on the disk geometry assumed in the analysis. For example, for the MERLIN data on V778 Cyg presented by Szczerba et al. (2006), Babkovskaia et al. (2006) applied a doubly warped disk model and derived a companion mass of $1.7 M_{\odot}$, which is significantly larger than the $0.06 M_{\odot}$ derived by Szczerba et al. (2006). This highlights, once again, the importance of long-term monitoring of the $\mathrm{H}_{2} \mathrm{O}$ masers in order to derive the properties of the disk and the secondary star.

\section{Conclusion}

Our VLBA observations of the $22 \mathrm{GHz} \mathrm{H}_{2} \mathrm{O}$ masers toward the silicate carbon star EU And have revealed that the masers are linearly aligned with a slight hint of an S-shaped structure, with the masers in SW redshifted and those in NE blueshifted. Such a spatio-kinematic structure can be interpreted either as an edge-on disk or an emerging jet. The $\mathrm{H}_{2} \mathrm{O}$ maser maps obtained at three epochs over 14 months show a little outward motion of 0.3 mas, but the outflow velocity of $\sim 4 \mathrm{~km} \mathrm{~s}^{-1}$ estimated from this outward motion is too low compared to those observed toward the water-fountain sources. This lends support to the disk interpretation, although some kind of outflow (possibly with a velocity much lower than in the water-fountain sources) cannot be entirely ruled out due to the uncertainty in the distance. The mid-infrared spectrum of EU And obtained with the Spitzer/IRS shows that the silicate emission is optically thin and emanates from sub-micron-sized grains, which suggests that the $\mathrm{H}_{2} \mathrm{O}$ masers originate in a circum-companion disk seen nearly edge-on. Furthermore, we unambiguously identified the $\mathrm{CO}_{2}$ features at $13-16 \mu \mathrm{m}$ in the Spitzer spectrum for the first time.

If we assume that the disk is in Keplerian motion, the mass of the putative secondary star is estimated to be $0.5-0.8 M_{\odot}$. However, given the remarkable variability of the water masers and the possible presence of fainter or more diffuse emission not detected by VLBA, the estimated mass should be regarded as a lower limit. Observations with a more compact, high-sensitivity, array (e.g., the VLA or MERLIN) might be useful in characterizing any such extended emission. Future VLBA observations will be necessary to put stronger constraints on the geometry of the disk and the properties of the secondary star. Astrometric monitoring with the VLBA should also provide more accurate estimates of the parallax and proper motion of EU And, thus improving our understanding of the three-dimensional structure of the oxygen-rich gas surrounding the system.

\section{References}

Babkovskaia, N., Poutanen, J., Richards, A. M. S., \& Szczerba, R. 2006, MNRAS, 370.1921

Barnbaum, C., Morris, M., Likkel, L., \& Kastner, J. 1991, A\&A, 251, 79

Boboltz, D. A., \& Marvel, K. B., 2007, ApJ, 665, 680

Colomer, F., Reid, M. J., Menten, K. M., \& Bujarrabal, V. 2000, A\&A, 355, 979

Deroo, P., Van Winckel, H., Verhoelst, T., et al. 2007, A\&A, 467, 1093

Engels, D. 1994, A\&A, 285, 497

Fey, A. L., Ma, C., Arias, E. F., et al. 2004, AJ, 127, 3587

Higdon, S. J. U., Devost, D., Higdon, J. L., et al. 2004, PASP, 116, 975

Houck, J., Roellig, T., van Cleve, J., et al. 2004, ApJS, 154, 18

Imai, H., Obara, K., Diamond, P. J., Omodaka, T., \& Sasao, T. 2002, Nature, 417, 829

Ireland, M., Monnier, J. D., Tuthill, P. G., et al. 2007, ApJ, 662, 651

Izumiura, H. 2003, Ap\&SS, 283, 189

Izumiura, H., Noguchi, K., Aoki, W., et al. 2007, ApJ, submitted

Jura, M., \& Kahane, C. 1999, ApJ, 521, 309

Little-Marenin, I. R. 1986, ApJ, 307, L15

Little-Marenin, I. R., Benson, P. J., \& Little, S. J. 1987, in Cool Stars, Stellar Systems, and the Sun, ed. J. L. Linsky, \& R. E. Stencel (Berlin: SpringerVerlag) 396

Little-Marenin, I. R., Benson, P. J., \& Dickinson, D. F. 1988, ApJ, 330, 828

Lloyd-Evans, T. 1990, MNRAS, 243, 336

Morris, M. 1987, PASP, 99, 1115

Nakada, Y., Izumiura, H., Onaka, T., et al. 1987, ApJ, 323, L77

Nakada, Y., Deguchi, S., \& Forster, J. R. 1988, A\&A, 193, L13

Ohnaka, K., \& Tsuji, T. 1999, A\&A, 345, 233

Ohnaka, K., Driebe, T., Hofmann, K.-H., et al. 2006, A\&A, 445, 1015

Pestalozzi, M., Elitzur, M., Conway, J. E., \& Booth, R. S. 2004, ApJ, 603, L113

Rothman, L. S., Jacquemart, D., Barbe, A., et al. 2005, JQSRT, 96, 139

Szczerba, R., Szymczak, M., Babkovskaia, N., et al. 2006, A\&A, 452, 561

Werner M. W., Roellig, T. L., Low, F. J., et al. 2004, ApJS, 154, 1

Willems, F., \& de Jong, T. 1986, ApJ, 309, L39

Yamamura, I., Dominik, C., de Jong, T., Waters, L. B. F. M., \& Molster, F. J. 2000, A\&A, 363, 629

Zacharias, N., Monet, D. G., Levine, S. E., et al. 2003, NOMAD Catalog

Zacharias, N., Urban, S. E., Zacharias, M. I., et al. 2004, AJ, 127, 3043 